\title{
Using low-cost sensors and citizen science to examine air quality
}

Air pollution is often a direct
cause of poor health. Although
useful, governmental air quality
recording sometimes lacks
spatial coverage. By harnessing
the power of modern day's
'digital residents, 'citizen science
projects can be easily employed
to continuously gather vast
quantities of data. Dr Andrew
May and colleagues from
The Ohio State University are
teaming up with teachers and
students across the Hilliard City
School District (HCSD) and other
partners to deploy low-cost air
quality sensors throughout the
Central Ohio (USA) region. They
aim to odentify areas for much
needed air quality control, whilst
integrating scientific learning
within the HCSD curriculum.

within the HCSD curriculum. espite many initiatives and efforts USA), Dublin City Schools (Dublin, Ohio, espite many initiatives and efforts
to reduce tailpipe emissions from motor vehicles, traffic-related
mated pollution (TRAP) remains a problem TRAP is full of toxic substances such as carbon monoxide (CO), nitrogen dioxide $\left(\mathrm{NO}_{2}\right)$, particulate matter $(\mathrm{PM})$, benzene, 13-butadiene, formaldehyde, acrolein, and acetaldehyde in addition to precursors for ozone $\left(\mathrm{O}_{3}\right)$ production. The long-term detriment of exposure to these pollutants on human health is well known, increasing risk of respiratory and cardiovascular disease, especially among children, elderly, and pregnant women.

In addition, areas of poor air quality are generally also regions of a lower socioeconomic status. Low-income housing has historically been built close to areas of pollution such as industrial point sources or high-traffic roadways. To make matters worse, the inhabitants of these areas are often less well equipped to de with the consequences of this pollution,

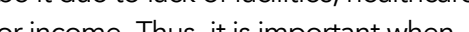
examining air quality to ensure data is collected over a gradient of wealth land-use, and demographics. Dr Andrew May and colleagues from The Ohio State University are teaming up with teachers and students across the Hilliard City USA), and Worthington City Schools (Worthington, Ohio, USA) to address this very issue in order to provide a reliable map of local air quality. Potential uses of these maps include alerting policy flow, identifying area for greater scruting for air quality regulation, and informing susceptible populations about areas to avoid on poor air quality days.

\section{LOW-COST REMOTE SENSORS} the measurement of air quality urbanised areas is the large variability in environments, and the low spatial resolution at which this occurs. For example, there could be a multi-lane highway adjacent to a large green space. One sensor across this area will only capture an average which would not cevalld for elher land-use type. This is predominantly the current issue in Frank Protection located to the northern and eastern portions of the county thus missing a substantial portion of the county entirely (including the 'downtown' urban core) as well as any changes in air quality within 'micro-environments' between the monitoring sites. This is particularly a School District (HCSD; Hilliard, Ohio, problem since data from the Center for

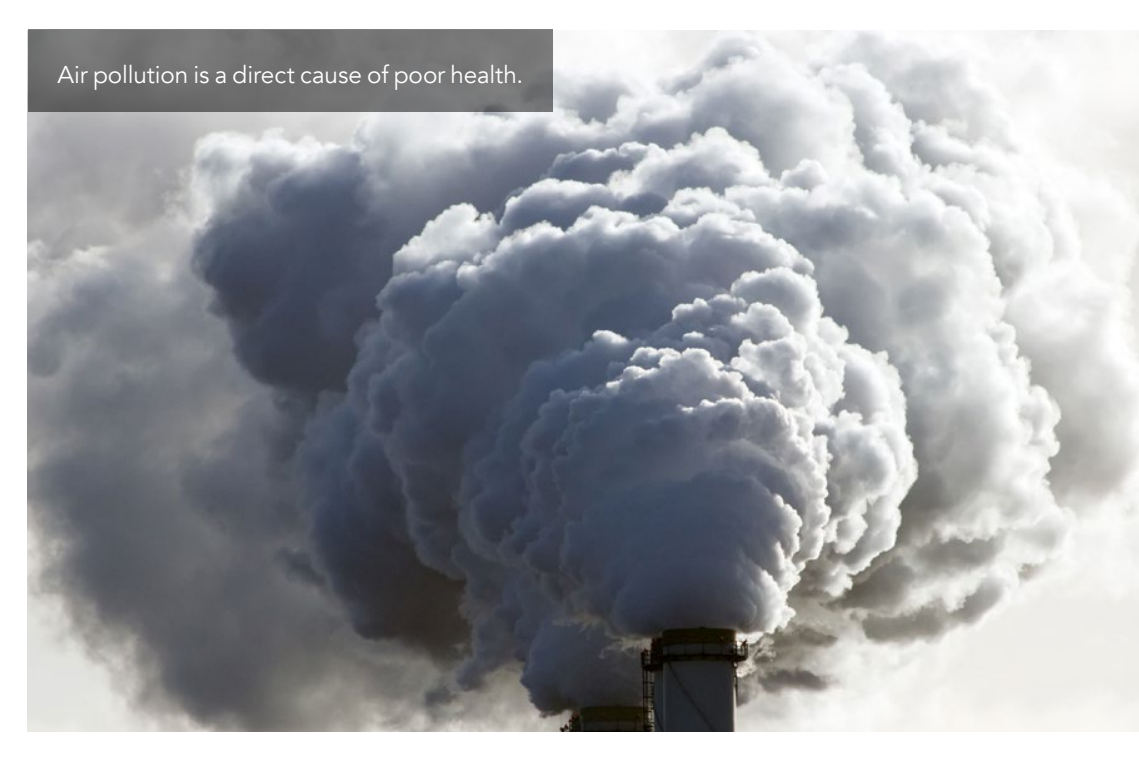

Disease Control's Behavioral Risk Factor Surveillance System Survey (BRFSS for short) has shown that asthma incidence and the highest prevalence tends to be in the central and southern portions of the county Therefore the data that the existing monitors provide may notbe representative of actual air quality with ity within

The initiative outlined by project investigators (PIs) Dr Andy May and Dr Ayaz Hyder aims to solve this problem through harnessing the power of citizen science, combined with the everadvancing world of low-costs sensors and cloud-based technologies. US EPA monitoring

equipment costs hundreds 作 and will be extremely power of a cost-effective 'sensing network' that can be widely'sentribut of 'off the shelf' sensor components, three cohorts of Grade 12 students have worked with the Pls to construct a small, low-cost, low-maintenance remote sensor packag which operate semi-autonomously with connectivity through a Wi-Fi network. These 'TRAP monitors' contain sensors that measure $\mathrm{CO}, \mathrm{NO}_{2}, \mathrm{O}_{3}+\mathrm{NO}_{2}, \mathrm{PM}_{t}$ and relative humidity/temperature and are attached to a Raspbery $\mathrm{P} 3 \mathrm{~s}$ singleboard computer for data acquistion and tansmission to the cloud. These devices require some background knowledge prigital electronics and computer programming (e.g., Python) to fabic

information is being taught to the HCSD high school students directly involved working to resolve via a solar panel unit curriculum at their school.

Wireless sensors of this nature do not come without their own problem Weatherproofing and battery life are practical challenges which the students (under the guidance of the PIs) are actively and full weatherproof housing for each device. Low-cost sensors may also be subject to bias and 'baseline signal drift' when compared with US EPA monitoring technologies, there are ways to account
for these issues including recalibration ...harnessing the power of citizen science, combined with the ever- $\quad \begin{aligned} & \text { The amount of data } \\ & \text { received has a huge }\end{aligned}$ and also covers a wide of the sensors or the implementation of data analytics tools.

\section{MEASUREMENTS AND DATA} Initially, the devices undergo a calibration procedure at The Ohio State University
using reference instrumentation that is similar to those deployed by the US EPA. This enables the translation of the sensors' raw output signal to a pollutant concentration. Once returned to the schools, students are trained on the basic Python code, and work together to deploy the sensors in an area with suitable Wi ficonnectivity. Around ten sensors will be deployed at various schools across the and socioeconomic status. accessed for analysis. The data is the

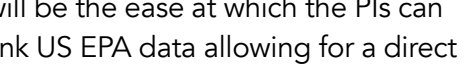
comparison assessm

Children and the elderly are especially
susceptible to poorairaculilyty potential for analysis breadth of scientific

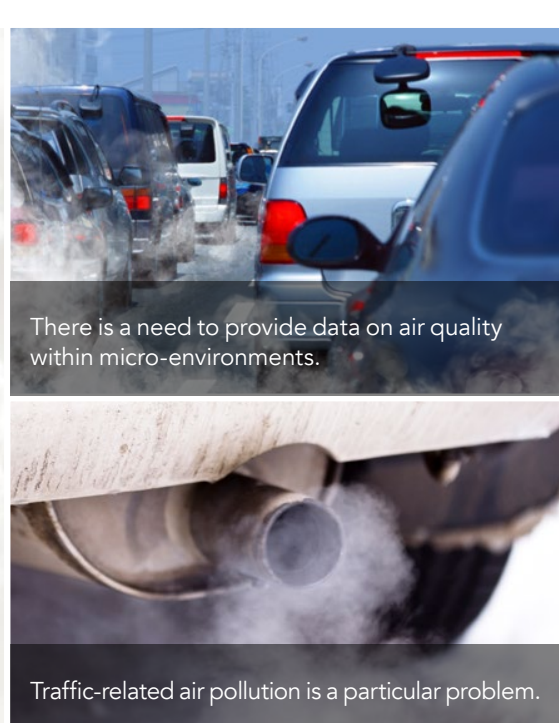

The primary goal is to determine the quality of recordings across time. into the 'cloud' specificlly Google's FireBase, where it can be centrally drawn from the cloud into a script written in the $\mathrm{R}$ programming language that enables data visualisation via a website using Shiny $R$ (currently in beta development). This is of course after any necessary calibration and wits to the cloud based approach will be the ease at which the Pls can comparison assessment visualisations will be maps of air quality across the HCSD for each pollutant, and the levels of each pollutant at various

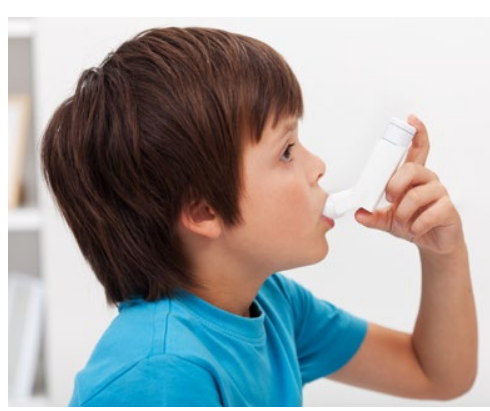



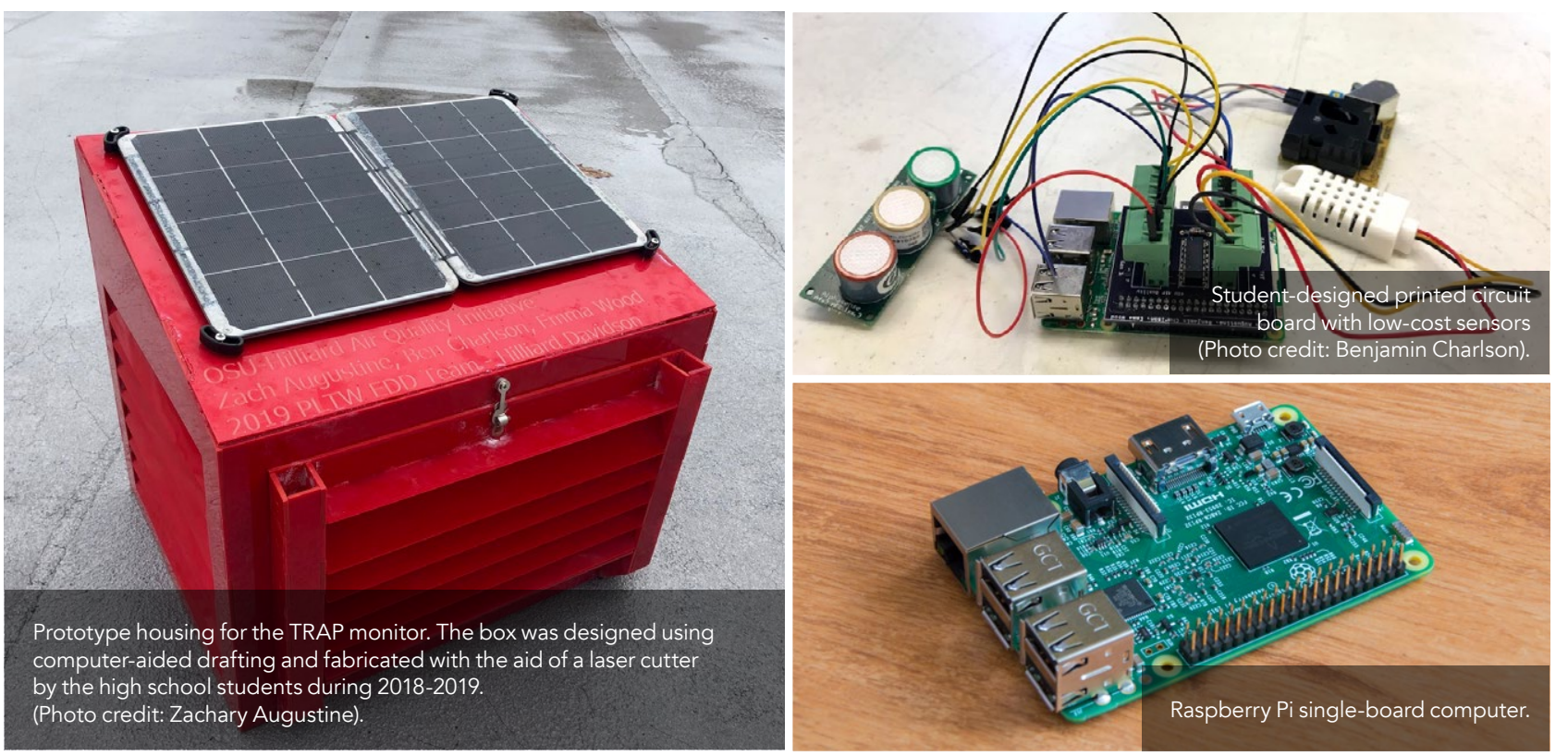

school sites. These data can be readily compared to data regarding traffic land-use, temporal pacterns, and also comparison against the US EPA's data.

of their peers in introductory engineering courses at university.

In order to cover the HCSD $\left(160 \mathrm{~km}^{2}\right)$ there will be ten sensing stations deploy across school buildings in the HCSD area, and these will require upkeep

CITIZEN SCIENCE AND EDUCATION This project really kills two birds with one stone. Not only will the new sensors provide important long-term temporal and spatial data, but the Pls are also working alongside schools in the HCSD to integrate science into the curricula. The students collaborating on the HCSD initiative use this as their project in an Engineering Design and engineering public helth, environmenta science, and statistics. They have gained first-hand experience on the iterative nature of this process, since the second year had the previous year's work and made improvements, and the third yea has improved on the first two years. The current team of students identified for the fourth year will continue to build on these efforts.

Furthermore, the initiative increases links between universities and schools, allowing students to contact and communicate with local experts, and take part in large scale scientic research otherwise unneachable until further education. Past students have indicated that participating in this project

Its low-cost, citizen science blueprint spreads the workloar cross institutions whilst integrating education and STEM themes.

fabrication and deployment step, schools across Columbus OH (USA) will use the sensors as subjects for teaching data this teaching, they can easily chack on the status of the sensors and report back data or damages (including localised warnings for asthmatics or other sen groups), truly embracing the power of citizen science.

A further benefit of integrating the HCSD community comes through the collaboration of committees inside education such as the HCSD School Board, and organisations outside of education, such as the City of Hilliard's Environmental Sustainability Commissi Students have presented progress presentations to these groups, which can lead to tangible actions regarding towards public transpools, a dive public awareness on the problems associated with air pollution.

This work has also led to other collaborations centered on education. Dr May and Dr Hyder have recently begun work with Dublin City Schools' merald Campus' Engineering Academy to integrate a similar curriculum, and Dr M has been working with the Columbus Public Health Department and Worthington City Schools will provide localised dat for that to explore in the classroom.

This project ticks two final important continuous imp data wil be of monitoring of air quality in Columbus $\mathrm{OH}$. If real-world changes are made the sensors will be vital to understanding if these changes are successful. Secondly, this initiative has the potential to be expanded across cities across the US, and the project template could be used worldwide. Although other groups are deploying low-cost sensors in urban areas what sets this project apart is the strong collaboration with local high schools. This low-cost, citizen science blueprint spreads the workload across and STEM them the Hopeng education and STEM themes. Hopefly it won't be long until we see the TRAP reeling in data

\section{Behind the Research}

(@) Dr Andrew May

E: may.561@osu.edu T: +1-614-688-1206 W: W.osu.edu/may.561

Detail

2070 Neil Ave

Hitchcock Hall Room 470

Columbus, Ohio 43085

United State of America

Bio

DrAndrew May is an Assistant Professor in Environmental Engineering at The Ohio State University. Aside from the work described here, his research interests are related environmental quality.

\section{Funding}

National Science Foundation (USA) Grant \#1645226: Ohio

\section{Collaborators}

- DrAyaz Hyder, College of Public Health at The Ohio State - University (Columbus, Ohio, USA)

- Hiliard City School District, in particular Mr Rusty Herring (Hilliard, Ohio, USA)

(Dy of Dublin, Uhio/Dublin City Schools

(Dublin, Ohio, USA)

- Rick Hicks, Columbus Public Health Department

(Columbus, Ohio, USA)

- Worthington City Schools (Worthington, OH, USA)
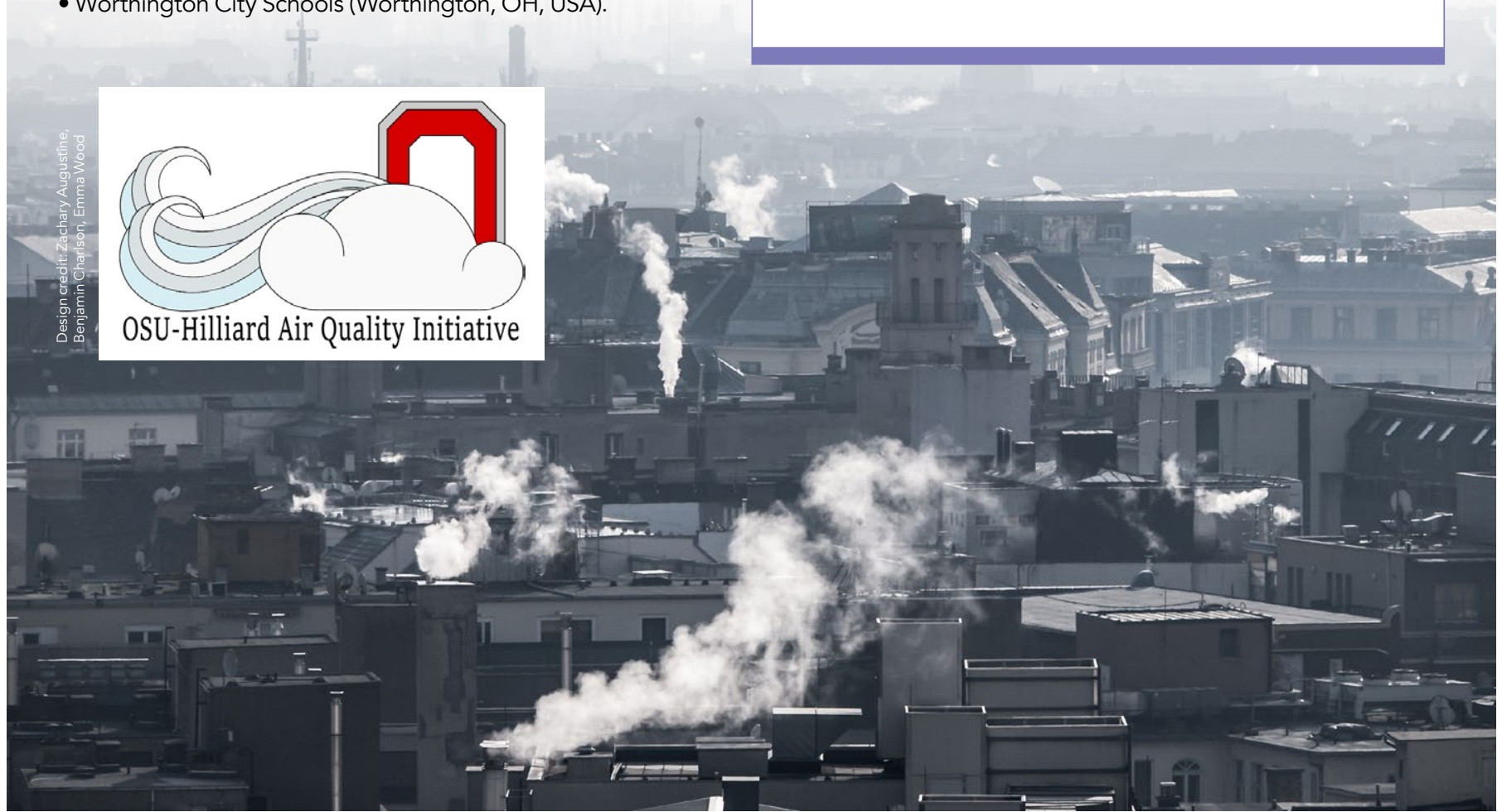

Research Objectives

The May research group focuses on air quality, a highly inter-disciplinary topic with much scope for collaboration.

\section{Personal Response}

What advice would you give to other researchers about Whaject?

II 1. Start early! We were very lucky to find some teachers and school districts who were willing collaborators fic
the outset, but this may not always be the case.

2. Temper your expectations. At this point, we only have limited data collection with HCSD. This has been integrated into a course, so there are other educational objectives that the students must meet. It also took a while to fully iteration on the previous efforts during each subsequent the students with a model for how to do everything but and engineering design on their own is arguably better year. We could address this issue by explicitly providing allowing them to struggle through the problem-solving 